\title{
Analysis of hold-up and grinding pressure in a spiral jet mill using CFD-DEM
}

\author{
Lewis Scott, Antonia Borissova, Alan Burns and Mojtaba Ghadiri* \\ School of Chemical and Process Engineering, The University of Leeds, UK
}

\begin{abstract}
A spiral jet mill was simulated using Discrete Element Method modelling and Computational Fluid Dynamics. The particle behaviour and fluid motion were analysed as a function of hold-up and grinding pressure. Particle collision energy was predicted to be prevalent along the bed surface and in front of the grinding jets, as shown through the collision data recorded. The bed itself affects the fluid flow field, as momentum is transferred to the particles. Increasing the grinding pressure does not result in a proportional increase in the kinetic energy of the particle system, as the high pressure jets begin to penetrate the bed with greater ease. The particle bed moves as 'plug-flow', with the layers of the bed closest to chamber wall.
\end{abstract}

\section{Introduction}

The spiral jet mill is the equipment of choice in many industries that require fine powders. The mill has no moving parts, which minimise both contamination and maintenance. Particle breakage in the mill is autogenous, as it is the result of inter-particle and particle-wall collisions. A series of jets angled around the wall of the milling chamber form an internal vortex, which accelerates the particles around the chamber [1]. The particles then escape through an opening at the centre of the mill, once they have sufficiently decreased in size. Due to particle breakage and size classification occurring within the same chamber, subtle changes in either will alter both the fluid flow field and particle behaviour.

During operation of the mill, a particle bed forms around the outer wall of the milling chamber due to the centrifugal action induced by the grinding jets. High strain rates are experienced by the particles, as the layers of the bed shear across one another [2]. Particles also experience high collisional energies, as they are ejected from the bed and impact on return [3]. Therefore, particle size reduction is the result of both chipping and fragmentation in the mill [4].

Grinding conditions in the spiral jet mill have been extensively studied, with the consensus agreeing that particle feed rate and grinding pressure are the two most influential parameters [1,5-9]. In general, increasing the grinding pressure results in a finer particle size, however, the final product size does not scale proportionally with pressure [10]. Small decreases in the grinding pressure during operation can widen the product size distribution, whereas significantly reducing the grinding pressure will destabilise the internal vortex [6,11]. Müller et al. [6] investigated the role of hold-up and residence time using a radioactive tracer. They found that hold-up decreased as grinding pressure was increased and derived a cut-size function with hold-up

*Corresponding author: M.Ghadiri@leeds.ac.uk

A video is available at https://doi.org/10.48448/np26-0710

(C) The Authors, published by EDP Sciences. This is an open access article distributed under the terms of the Creative Commons Attribution License 4.0

(http://creativecommons.org/licenses/by/4.0/). as a parameter. MacDonald et al. [12] derived a series of analytical equations, which included hold-up, to also predict the product cut-size. Luczak [13] and Luczak et al. [2] analysed the particle flow patterns as a function of grinding pressure using particle velocimetry. They found that size reduction intensity is prevalent in the top layer of the particle bed; also shown by Scott et al [3] using CFD-DEM simulations. However, they also showed grinding is prevalent on the frontside of the jet (relative to the particle), something in contrast to Kürten and Rumpf [14], but in agreement with Scott et al [3].

CFD-DEM simulations of the spiral jet mill have been limited in the past due to computational power required [11,15-17]. Rodnianski et al. [18] and Bnà et al [19] used CFD-only and one-way coupled CFD-DEM to simulated the mill, respectively. However, unlike two-way coupling, or higher order schemes, one-way coupling and CFD-only simulations are unable to capture the dampening effect of the particle bed on the fluid flow field.

Here, the spiral jet mill is studied using a four-way coupled CFD-DEM scheme [20-23] to analyse the effect of grinding pressure and hold-up on particle behaviour and the fluid flow field. The basis for the mill is the Hosokawa Micron AS-50 (Runcorn, UK).

\section{Method}

Using CAD, an in-house Hosokawa Micron AS-50 was drawn, as shown in Figure 1. The drawing included both the annular manifold and the hopper section to improve the fluid flow field calculation. The red box defines the simulation domain in $\mathrm{EDEM}^{\mathrm{TM}}$ and encapsulates the entire volume of the milling chamber, including the vortex finder. The milling chamber is $50 \mathrm{~mm}$ in diameter with four equally spaced, angled jets to induce the vortex. $\mathrm{EDEM}^{\mathrm{TM}}$ (Altair, UK) was used to calculate the particle behaviour and FLUENT 18.1 (ANSYS, USA) was used to predict the fluid flow field. 


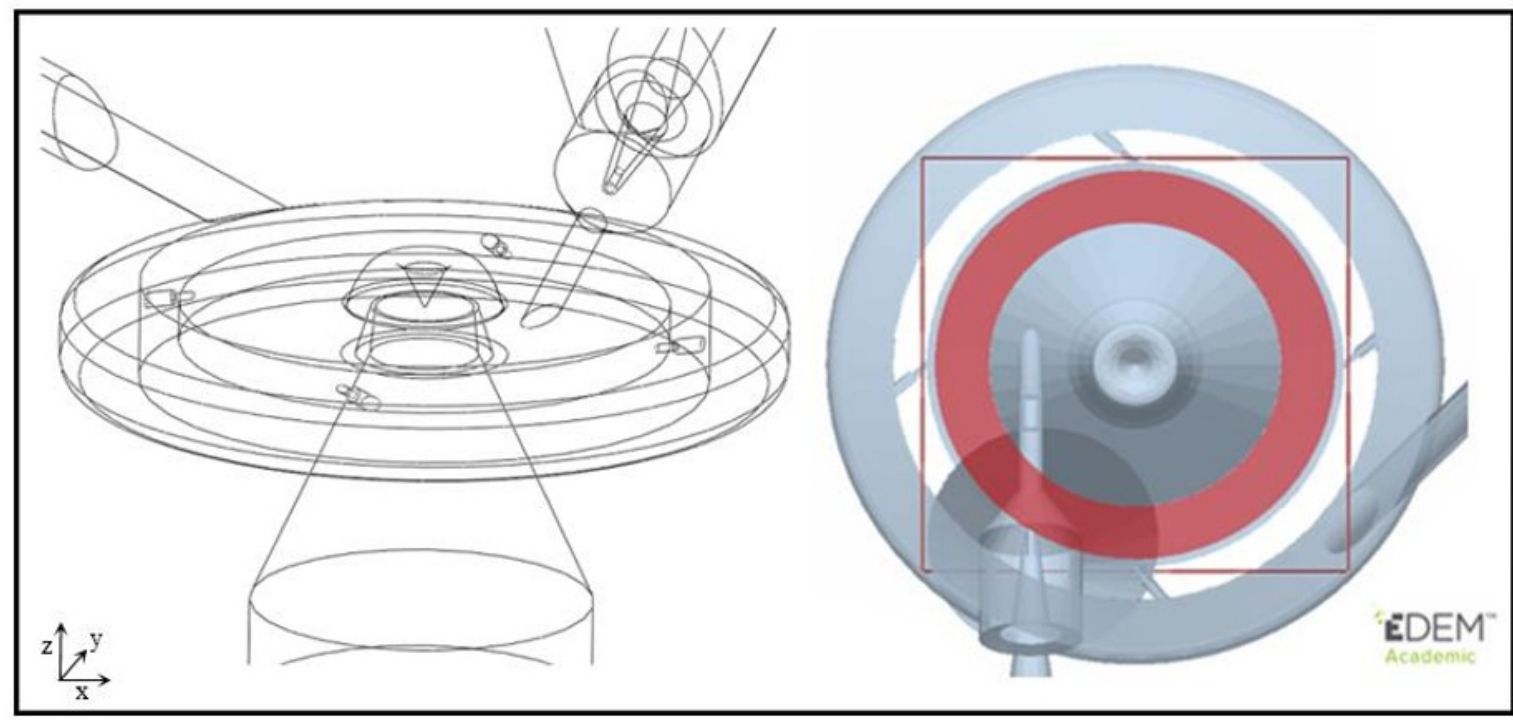

Fig 1: In-house drawing of the Hosokawa Micron AS-50 (left). Simulation domain in EDEM ${ }^{\mathrm{TM}}$ with simulation factory highlighted in red (right)

The $k-\varepsilon$-RNG turbulence model and Morsi and Alexander drag model [24] were used in the simulation. The settings for mass loadings and fluid conditions are given in Tables $1 \& 2$. The particle parameters are given in Table 3. Hertz-Mindlin contact model was used throughout. A four-way coupled scheme adopted to capture all particle-fluid interactions; i.e. fluid damping and particle-particle collisions. In both studies, the particles were added directly into the chamber by way of the particle factory highlighted in red. A mesh independence study was carried out prior to this study to determine the validity of the CFD mesh used. Following Norouzi et al. [25], a CFD time-step no larger than thirty DEM time-steps was implemented. A DEM time-step lower than $25 \%$ the Rayleigh's time-step was also maintained throughout each case.

Table 1: Hold-up study parameters

\begin{tabular}{|c|c|c|}
\hline Mass loadings $(\mathrm{g})$ & $0.4,0.8,1.2,1.6,2.0$ \\
\hline \multirow{3}{*}{$\begin{array}{c}\text { Particle diameter }(\mu \mathrm{m}) \text { and } \\
\text { size distribution }(\mathrm{wt} \%)\end{array}$} & 300 & 20 \\
\cline { 2 - 3 } & 240 & 40 \\
\cline { 2 - 3 } & 200 & 20 \\
\cline { 2 - 3 } & 160 & 20 \\
\cline { 2 - 3 } Number loading for each \\
\cline { 2 - 3 } mass loading $(\mathrm{g})$ & 0.4 & 54321 \\
\cline { 2 - 3 } & 1.2 & 111005 \\
\cline { 2 - 3 } & 1.6 & 224206 \\
\cline { 2 - 3 } & 2.0 & 280366 \\
\hline Fluid feed pressure $(\mathrm{kPa})$ & \multicolumn{2}{|c|}{3200} \\
\hline Fluid grinding Pressure \\
$(\mathrm{kPa})$
\end{tabular}

\subsection{Hold-up study}

Keeping the grinding pressure constant at $3 \mathrm{barg}$, the mass of particles in the mill is increased for each case. The addition rate was set for each case, so that the required mass would be added within $0.01 \mathrm{~s}$. A period of time, around $0.03 \mathrm{~s}$, was then allowed for the particle bed to reach a pseudo-steady-state. After this time, there is no significant variation in the total kinetic energy of the particle system. Collisional and velocity data were then recorded, up until $0.1 \mathrm{~s}$ when each simulation was halted.

Table 2: Pressure study parameters

\begin{tabular}{|c|c|c|c|c|}
\hline $\begin{array}{c}\text { Fluid Feed } \\
\text { Pressure (kPa) }\end{array}$ & 300 & 400 & 500 & 600 \\
\hline $\begin{array}{c}\text { Grinding } \\
\text { Pressure (kPa) }\end{array}$ & 200 & 300 & 400 & 500 \\
\hline $\begin{array}{c}\text { Mass } \\
\text { Loading(g) }\end{array}$ & \multicolumn{4}{|c|}{ Particle number } \\
\hline 0.4 & 61857 & 61869 & 61701 & 61884 \\
\hline 0.8 & 123684 & 123481 & 123467 & 123404 \\
\hline 1.2 & 184576 & 184958 & 184796 & 184763 \\
\hline 1.6 & 246368 & 246025 & 246248 & 245892 \\
\hline 2 & 310191 & 308539 & 307964 & 307393 \\
\hline
\end{tabular}

Table 3: Particle properties used in all simulations

\begin{tabular}{|l|c|}
\hline Particle parameters & value \\
\hline Density, kg/m $\mathrm{m}^{3}$ & 1500 \\
Shear modulus, MPa & 10 \\
Poisson's ratio & 0.25 \\
Coefficient of restitution & 0.5 \\
Coefficient of static friction & 0.5 \\
Coefficient of rolling friction & 0.01 \\
DEM time step, $\mu \mathrm{s}$ & $0.4-1.0$ \\
\hline
\end{tabular}

\subsection{Pressure study}

A random particle size distribution in the range of $80-$ $200 \mu \mathrm{m}$ was used. Each mass loading was then repeated for the four absolute grinding pressures, i.e. 200, 300, $400,500 \mathrm{kPa}$. 


\section{Results}

\subsection{Hold-up Study Discussion}

\subsubsection{Fluid field}

Three distinct regions developed in the milling chamber after the particle bed formed; a slow moving dense particle bed region and a lean phase fast moving region next to the classifier wall. As bed depth increased, the jet penetration depth decreased, due to dampening effect of the particles. It was also found that as mass loading increased, the velocity surrounding the classifier decreased. There was no direct dampening of the fluid flow field around the classifier, as the particles could not travel that far. Instead the fluid flow field was indirectly dampened by the presence of the bed, as more energy is transferred to accelerate the particles and stabilise the vortex.

\subsubsection{Particle behaviour}

The particles are added to the simulation with zero velocity. They then quickly form the particle bed, as they accelerate around the chamber. It can be seen in Figure 2 that total energy of the particle systems reach a similar asymptotic value of around $7 \mathrm{~mJ}$. It remains unclear why this happens, however, the only source of energy comes from the feed and grinding jets.

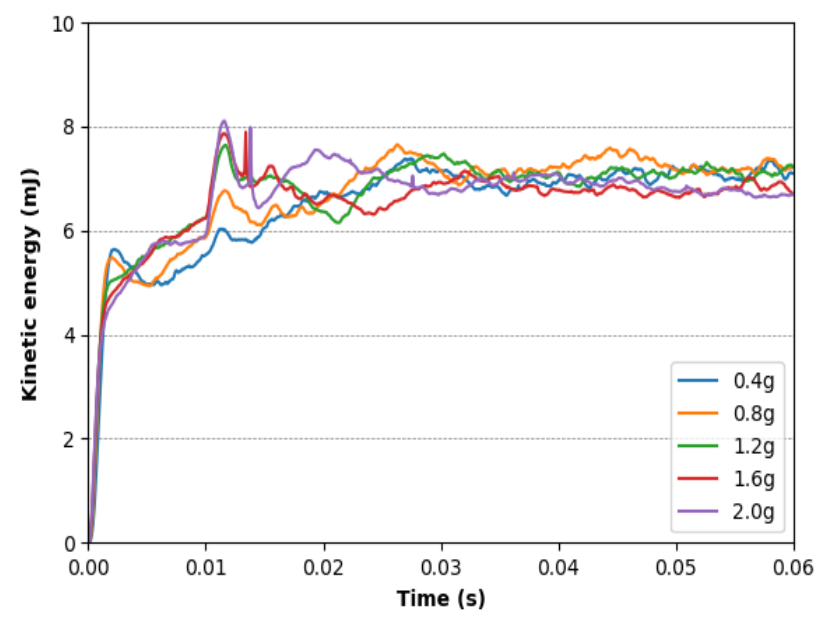

Fig 2: Kinetic energy for each particle system

Overall, the highest particle velocity was recorded in the $0.4 \mathrm{~g}$ loading case. Compared to the other cases, fewer particles are accelerated and collisions occur less frequently. This allows the particles to achieve a higher velocities for lower loadings. Figure 3 displays a radial velocity profile of a single slice taken through the bed. The profiles span from $0-10 \mathrm{~mm}$ from the chamber wall and agree well with the experimental velocimetry work of Luczak [2]. As the material in a bed increases, the velocity gradient decreases first due to the presence of the bed before rapidly increasing. Interestingly, the particles in the $1.6 \mathrm{~g}$ and $2.0 \mathrm{~g}$ cases display "plug-flow" behaviour close to the wall.

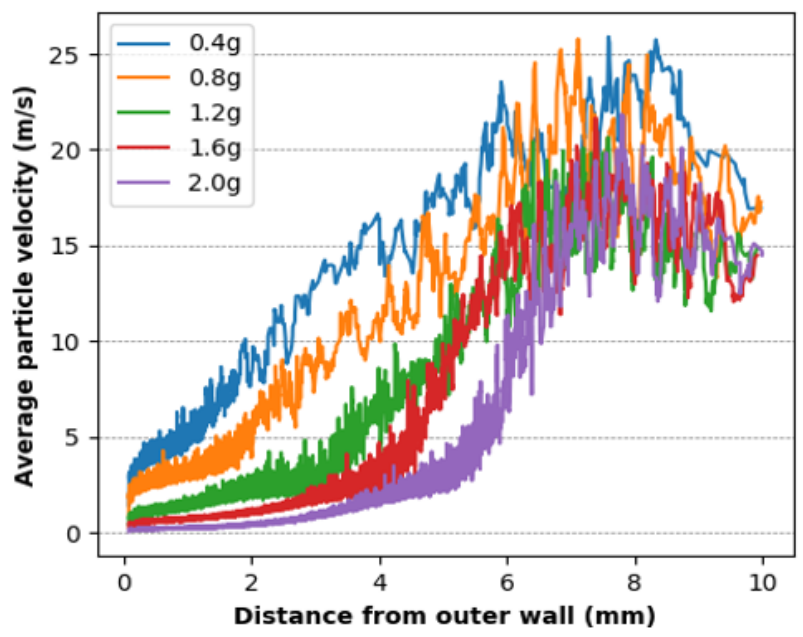

Fig 3: Radial velocity profile from the outer wall

\subsubsection{Particle collision intensity}

As the mass loading increases within the mill, the overall power dissipated increases from $4.05 \mathrm{~W}$ to 4.65 W. Particle collision frequency also increases. However, the average amount of energy dissipated during a collision decreases due to the reduced average particle velocity. In agreement with the work of Luczak et al. [2], the highest rate of energy dissipated occurs at the bed surface where the rate of shearing is also at its highest. There is also a high rate of energy dissipation in front of the jets, as the particles collide due to the sudden change in direction.

\subsection{Pressure study}

As the pressure increases, the average particle velocity also increases and the particles closest to the bed surface shear at a higher rate. However, at the highest pressure, the particles closest to the wall in the $2.0 \mathrm{~g}$ case exhibit plug-flow behaviour. This is because the grinding jets penetrate through the bed with greater ease as pressure increases. The energy available to be transferred to the particles is therefore limited, as the air may only further transfer energy to the particles along the face of the bed before being directed out of the mill, due to the low downstream pressure.

Due to the limited exchange of the energy with the air, the kinetic energy of the system particle system does not increase proportionally with grinding pressure. This leads to the dissipated energy of the particle system to only increase from $0.17 \mathrm{~mJ}$ to $0.6 \mathrm{~mJ}$, with an increase of grinding pressure from 3 barg to 6 barg; as shown in Figure 4. Ramanujam and Venkateswarlu [5] and Kürten \& Rumpf [10] report a similar relationship, as they both noted that increasing pressure does not lead to a proportional decrease in the final product size. However, the under-utilisation of the fluid energy as the jets penetrates through the bed, provides a different reasoning as to why both groups found this result. 


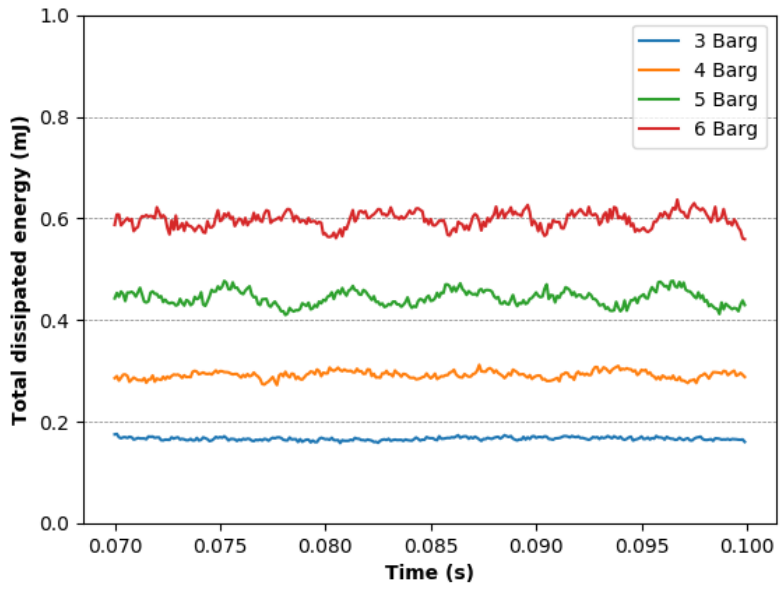

Fig 4: Energy dissipated through particle collisions for the $1.2 \mathrm{~g}$ loading case

\section{Conclusions}

Both hold-up and pressure effects were investigated in the spiral jet mill using a 4-way coupled CFD-DEM simulation. The amount of material held in the mill indirectly influences the air velocity around the classifier, as kinetic energy is transferred to the particle bed. High amounts of dissipated energy were recorded along the bed surface and in front of the grinding jets, and therefore, a prevalent areas of the mill where grinding is thought to take place.

Increasing the particle mass leads to a reduction in the average particle velocity. However, increasing the number of particles also leads to an increase in the collision frequency, and therefore, the rate of energy dissipation stays almost constant for a particular grinding pressure.

Increasing the grinding pressure leads to an increase in the kinetic energy and energy dissipated rate of the particle system. However, this increase does not scale proportional with pressure. It is concluded that the low increase in energy is due to the under-utilisation of the fluid energy available as the fluid jets penetrate through the particle bed.

This project is supported by the Engineering and Physical Sciences Research Council, UK, through the Centre of Doctoral Training for Complex Particulate Products and Processes (CDT $\mathrm{CP}^{3}$ ) (EPSRC Grant EP/L015285/1), which forms a part of the doctoral studies in collaboration with AstraZeneca Ltd. The authors gratefully acknowledge their support and would also like to especially thank Ian Gabbott and Catherine Hallam for their project co-ordination.

\section{References}

[1] R. Tuunila, L. Nyström, Miner. Eng. 11, 10891094 (1998)

[2] B. Luczak, R. Müller, C. Kessel, M. Ulbricht, H.J. Schultz, Powder Technol. 342, 108-117 (2019)

[3] L. Scott, A. Borissova, A. Burns, M. Ghadiri, Powder Technol. 377, 233-243 (2021)

[4] S. Dogbe, M. Ghadiri, A. Hassanpour, C. Hare, D.
Wilson, R. Storey, I. Crosley, EPJ Web Conf. 140, 7-10 (2017)

[5] M. Ramanujam, D. Venkateswarlu, Powder Technol. 3, 92-101 (1969)

[6] F. Müller, R. Polke, G. Schädel, Int. J. Miner. Process. 44-45, 315-326 (1996)

[7] S. Palaniandy, K.A.M. Azizli, H. Hussin, S.F.S. Hashim, Miner. Eng. 21, 380-388 (2008)

[8] P. Nair, M. Ramanujam, Adv. Powder Technol. 3, 273-284 (1992)

[9] A. Katz, H. Kalman, Part. Part. Syst. Charact. 24, 332-338 (2007)

[10] H. Kürten, H. Rumpf, Chemie Ing. Tech. 38 331342 (1966)

[11] T. Brosh, H. Kalman, A. Levy, I. Peyron, F. Ricard, Powder Technol. 257, 104-112 (2014)

[12] R. MacDonald, D. Rowe, E. Martin, L. Gorringe, Powder Technol. 299, 26-40 (2016)

[13] B. Luczak, Flow Conditions inside Spiral Jet Mills and Impact on Grinding Performance Dissertation Zur Erlangung Des Akademischen Grades Eines, Universitat Duisburg-Essen, (2018)

[14] H. Kürten, H. Rumpf, Chemie Ing. Tech. 38, 1187-1192 (1966)

[15] T. Han, H. Kalman, a. Levy, Part. Sci. Technol. 20, 325-340 (2002)

[16] S. Teng, P. Wang, Q. Zhang, C. Gogos, Powder Technol. 208, 684-693 (2011)

[17] S.C. Dogbe, Predictive Milling of Active Pharmaceutical Ingredients and Excipients, The University of Leeds, (2017)

[18] V. Rodnianski, N. Krakauer, K. Darwesh, A. Levy, H. Kalman, I. Peyron, F. Ricard, Powder Technol. 243, 110-119 (2013)

[19] S. Bnà, R. Ponzini, M. Cestari, C. Cavazzoni, C. Cottini, A. Benassi, Powder Technol. 364, 746773 (2020)

[20] S. Elghobashi. Applied Scientific Research, 48, 301-314 (1991)

[21] Y. Tsuji, T. Kawaguchi, T. Tanaka, Powder Technol. 77, 79-87 (1993)

[22] B.H. Xu, a. B. Yu, Chem. Eng. Sci. 52, 27852809 (1997)

[23] A. Di Renzo, F.P. Di Maio, R. Girimonte, B. Formisani, Powder Technol. 184, 214-223 (2008)

[24] S.A. Morsi, A.J. Alexander, J. Fluid Mech. 55, 193-208 (1972)

[25] H. Norouzi, R. Zarghami, R. Sotudeh-Gharebagh, N. Mostoufi, Coupled CFD-DEM Modeling, John Wiley \& Sons, Chichester, (2016) 